PROCEEDINGS OF THE

AMERICAN MATHEMATICAL SOCIETY

Volume 125, Number 8, August 1997, Pages 2265-2274

S 0002-9939(97)03851-3

\title{
ON SUBALGEBRAS OF BOOLEAN INTERVAL ALGEBRAS
}

\author{
LUTZ HEINDORF
}

(Communicated by Andreas R. Blass)

\begin{abstract}
We prove that the following three conditions are necessary and sufficient for a Boolean algebra $A$ to be embeddable into an interval algebra.

(i) $A$ is generated by a subset $R$ such that $r \cdot s \in\{0, r, s\}$ for all $r, s \in R$.

(ii) $A$ has a complemented subalgebra lattice, where complements can be chosen in a monotone way.

(iii) $A$ is isomorphic to Clop X for a compact zero-dimensional topological semilattice $(X ; \cdot)$ such that $x \cdot y \cdot z \in\{x \cdot y, x \cdot z\}$ for all $x, y, z \in X$.
\end{abstract}

Boolean algebras that are generated by subchains, i.e. subsets that are linearly ordered under the Boolean partial order, were studied as early as 1939 by A. Mostowski and A. Tarski [9] and have received much attention ever since. Nowadays they are called interval algebras. All basic facts about them can be found in section 15 of [4].

For the sake of brevity we introduce the phrase subinterval algebra to express that a Boolean algebra can be embedded into an interval algebra. Subinterval algebras need not be interval algebras again, the easiest example being the subalgebra generated by an uncountable set of pairwise disjoint elements. Our aim is to give characterizations of subinterval algebras that refer only to the algebra itself.

The first of these is in terms of special sets of generators: each subinterval algebra is generated by a ramification set, i.e. a subset $R$ such that $r \leq s$ or $s \leq r$ or $r \cdot s=0$ for all $r, s \in R$. This is equivalent (cf. [5, Theorem 2.3]) to saying that subinterval algebras are what J. D. Monk called pseudo-tree algebras. The other direction being known already (cf. the very nice proof of [5, Theorem 3.1]), it follows that the class of Boolean pseudo-tree algebras coincides with the class of subinterval algebras. As a corollary one gets that the class of pseudo-tree algebras is closed under taking subalgebras.

This result answers a question posed by Koppelberg and Monk in [5] and also by van Douwen in [3]. In topological form the characterization was recently proved by S. Purisch [12], who deduced it from a result of J. Nikiel's [10, Theorem 2.1] about the embeddability of certain spaces into dendrons. The author wishes to thank J.D. Monk for making his work-out [8] of Purisch's proof available to him. For superatomic algebras the characterization is also known: in [2] R. Bonnet, M. Rubin and H. Si-Kaddour constructed ramification sets of generators with additional nice properties.

Received by the editors November 1, 1995 and, in revised form, March 11, 1996.

1991 Mathematics Subject Classification. Primary 06E05; Secondary 54F05.

(C)1997 American Mathematical Society 
Our second characterization of subinterval algebras will be categorical, i.e. it could (we leave the details to the interested reader) be expressed in terms of homomorphisms and their compositions only. Let us call a Boolean algebra $A$ (monotonically) retractive if for each proper ideal $I \subseteq A$ there exists a subalgebra $C_{I} \leq A$ such that $C_{I} \cap I=\{0\}, C_{I} \cup I$ generates $A$ (and $C_{I} \leq C_{J}$, whenever $\left.I \supseteq J\right)$.

We call the subalgebra $C_{I}$ a (monotonically chosen) retract of $A$ with respect to $I$. This is because the first two conditions amount to saying that $C_{I}$ contains exactly one member of each congruence class of $A$ modulo $I$. In still other words, $A$ is retractive iff each onto homomorphism $A \rightarrow B$ has a right inverse $B \rightarrow A$.

In [14] B. Rotman conjectured that the retractive Boolean algebras are exactly the subinterval algebras. This conjecture was settled by M. Rubin in [15], who proved that subinterval algebras are indeed retractive and constructed (under $\mathrm{CH}$ resp. $\diamond)$ several retractive Boolean algebras that are not subinterval algebras. Since then more such examples have been given, e.g. in [16] and [6]; none are known in ZFC. Let us also mention that no retractive Boolean algebra with a nonretractive subalgebra is known. Below we prove that a modified version of Rotman's conjecture is true: a Boolean algebra is monotonically retractive iff it is embeddable into an interval algebra.

Let us say that the Boolean algebra $A$ is (monotonically) subalgebra-complemented if for each subalgebra $B \leq A$ there exists a subalgebra $B^{c} \leq A$ such that $B^{c} \cap B=$ $\{0,1\}, B^{c} \cup B$ generates $A$ (and $B^{c} \leq C^{c}$, whenever $C \geq B$ ). The subalgebra $B^{c}$ will be called a (monotonically chosen) complement of $B$ with respect to $A$.

A look at both definitions yields at once that a subalgebra of $A$ is a retract with respect to $I$ iff it is a complement of the subalgebra generated by $I$. It follows that (monotonically) subalgebra-complemented Boolean algebras are (monotonically) retractive. The converse is not obvious. In the non-monotonic version it is not even true: S. Todorčević observed (unpublished, quoted in [1]) that one of Rubin's retractive algebras is not subalgebra-complemented. Using Rubin's methods, Todorčević has also shown that all subinterval algebras are subalgebra-complemented. Let us mention that there is no known example disproving the converse.

To sum up the discussion so far, here is our main result.

Theorem 1. The following conditions are equivalent for each Boolean algebra A.

(a) A can be embedded into an interval algebra.

(b) A is monotonically subalgebra-complemented.

(c) A is monotonically retractive.

(d) A has a ramification set of generators.

The proof will be cyclic. In the next section we modify Rubin's construction of retracts in such a way that it yields the implication $(a) \Rightarrow(b)$. In section 2 we prove $(c) \Rightarrow(d)$. As mentioned above, $(b) \Rightarrow(c)$ is obvious and $(d) \Rightarrow(a)$ already known. In section 3 we give an alternative direct proof of the implication $(a) \Rightarrow(d)$, which may be of independent interest. The final section briefly discusses the topological consequences of Theorem 1 and adds another equivalent condition, namely

(e) There is a multiplication - on the Stone space $X$ of $A$ making it a topological semilattice such that $x \cdot y \cdot z \in\{x \cdot y, x \cdot z\}$ for all $x, y, z \in X$.

Our notation is in accordance with [4], with the exception that we use $\vee, \wedge$, and - for the lattice-theoretic Boolean operations of join, meet and complementation and reserve + for symmetric difference: $a+b=(a \wedge-b) \vee(b \wedge-a)$. In connection 
with + , meets are sometimes called products and denoted by $\cdot$ instead of $\wedge$. Recall that each Boolean algebra is a ring with unit under this addition and multiplication. Moreover, $a+a=0$ for all $a$, which implies that the underlying additive group of a Boolean algebra can be regarded as a vector space over the field with two elements. If $M$ is a subset of a Boolean algebra $A$, we denote by $\langle M\rangle$ the subalgebra generated by $M$. Notice that $\langle M\rangle$ is also the smallest subring with unit contained in $A$.

Some arguments become more transparent if we represent a given Boolean algebra as Clop $X$, the Boolean algebra of all clopen (= closed and open) subsets of some compact and zero-dimensional topological space $X$. In this case we use the set-theoretic notation $\cap, \cup, \triangle, \emptyset$, and $X$ instead of $\wedge, \vee,+, 0$, and 1 .

\section{The CONSTRUCTION OF COMPLEMENTS}

In the whole section $A$ is a fixed Boolean algebra generated by a ramification set $K$, which is also fixed. In particular, $A$ may be an interval algebra and $K$ its generating chain. Without loss we assume $0,1 \notin K$. All our arguments rely more or less upon the following observation.

(1) If $L \subseteq K$, then each non-zero element of $\langle L\rangle$ can be written in the form $l_{1}+l_{2}+\cdots+l_{n}$, where $n \geq 1$ and all $l_{i}$ belong to $L \cup\{1\}$.

In other words, $L \cup\{1\}$ linearly spans $\langle L\rangle$. To see this, recall that for arbitrary $M \subseteq A$, the subalgebra $\langle M\rangle$ consists of 0 and all finite sums of products of elements of $M \cup\{1\}$. As $L$ is a (subset of a) ramification set, $L \cup\{0,1\}$ is closed under products, which yields the the desired representations.

We now describe two monotone constructions of complements. The first of them is very simple and apparently new, which (hopefully) justifies its inclusion in the paper. The second construction is a careful version of the method used by Rubin and Todorčević. The extra care yields 'better' complements and finally leads to a proof of the implication $(a) \Rightarrow(b)$.

In both cases we need a well-ordering of $K$, most conveniently given as a fixed enumeration by ordinals: $\left(k_{\alpha}\right)_{\alpha<\kappa}$. The first construction becomes more transparent if we put $A=C l o p X$ and use the following well-known fact.

(2) A subset $G$ of $A=C l o p X$ generates $A$ iff it separates the points of $X$, i.e. if for all $x \neq y$ in $X$ there exists some $g \in G$ such that $x \in g \Longleftrightarrow y \notin g$.

First CONSTRUCTION. Fact $(2)$ shows that for each pair $(x, y)$ of distinct points of $X$, there is a least ordinal $\alpha(x, y)<\kappa$ such that $k_{\alpha(x, y)}$ separates $x$ and $y$. For a given subalgebra $B \leq A$ we put

$$
G_{B}=\left\{k_{\alpha(x, y)}: x, y \in X ; B \text { does not separate } x \text { and } y\right\}
$$

and check that $\left\langle G_{B}\right\rangle$ is a monotonically chosen complement of $B$ with respect to $A=C \operatorname{lop} X$.

By construction, $B \cup G_{B}$ separates the points of $X$, so it generates $A$. Monotonicity is also clear, for, the bigger the subalgebra $B$, the more points it separates, the smaller $G_{B}$ becomes. To see that $B \cap\left\langle G_{B}\right\rangle=\{0,1\}$, we assume the contrary and consider some $b \in B \cap\left\langle G_{B}\right\rangle \backslash\{0,1\}$. Passing to $1+b$ if necessary, (1) yields a representation

$$
b=k_{\alpha\left(x_{1}, y_{1}\right)}+\cdots+k_{\alpha\left(x_{n}, y_{n}\right)},
$$

where $n \geq 1$ and, without loss, $\alpha\left(x_{1}, y_{1}\right)>\alpha\left(x_{2}, y_{2}\right)>\cdots>\alpha\left(x_{n}, y_{n}\right)$. But this implies

$$
k_{\alpha\left(x_{1}, y_{1}\right)}=b+k_{\alpha\left(x_{2}, y_{2}\right)}+\cdots+k_{\alpha\left(x_{n}, y_{n}\right)},
$$


which is impossible because $k_{\alpha\left(x_{1}, y_{1}\right)}$ separates $x_{1}$ and $y_{1}$, while no term on the right-hand side does. Indeed, the other $\alpha$ are too small and $k_{\alpha\left(x_{1}, y_{1}\right)} \in G_{B}$ implies that $b$ does not separate $x_{1}$ and $y_{1}$.

Second construction. Let $B \leq A$ be given. By induction on $\alpha \leq \kappa$ we define subsets $L_{\alpha}^{B}$ of $K$. We start with $L_{0}^{B}=\emptyset$ and put $L_{\beta}^{B}=\bigcup_{\alpha<\beta} L_{\alpha}^{B}$ for limit ordinals. In the successor step we assume that $L_{\alpha}^{B} \subseteq\left\{k_{\beta}: \beta<\alpha\right\}$ has been defined. If there are finitely many $k_{\beta_{1}}, \ldots, k_{\beta_{n}} \in L_{\alpha}^{B}$ such that $k_{\alpha}+k_{\beta_{1}}+\cdots+k_{\beta_{n}} \in B$, in particular, if $k_{\alpha} \in B$ (and $n=0$ ), then $L_{\alpha+1}^{B}=L_{\alpha}^{B}$. Otherwise $L_{\alpha+1}^{B}=L_{\alpha}^{B} \cup\left\{k_{\alpha}\right\}$.

Notice that, by construction,

(3) $k_{\alpha} \in L_{\kappa}^{B} \Longleftrightarrow k_{\alpha} \in L_{\alpha+1}^{B}$.

We are going to show that $B^{*}=\left\langle L_{\kappa}^{B}\right\rangle$ is a monotonically chosen complement of $B$. To prove

(4) $B^{*} \cap B=\{0,1\}$,

we assume the contrary and consider some $b \in B^{*} \cap B \backslash\{0,1\}$. Passing to $1+b$ if necessary, (1) yields a representation $b=k_{\beta_{1}}+\cdots+k_{\beta_{n}}$ with all $k_{\beta_{i}} \in L_{\kappa}^{B}$. Let $\beta_{m}$ be maximal among the $\beta_{i}$. Then, by construction, $k_{\beta_{m}} \notin L_{\beta_{m}+1}^{B}$, hence, by (3), $k_{\beta_{m}} \notin L_{\kappa}^{B}$, contradiction.

Next we need that $\left\langle B^{*} \cup B\right\rangle=A$. We do a little better and show $A=B^{*}+B=$ $\left\{b^{*}+b: b^{*} \in B^{*}\right.$ and $\left.b \in B\right\}$. By (1), it is clearly sufficient to express each $k_{\alpha}$ in the form $b^{*}+b$. This is trivial if $k_{\alpha} \in L_{\kappa}^{B}$. But otherwise $k_{\alpha} \notin L_{\alpha+1}^{B}$ and there is some finite (possibly zero) sum $f \in\left\langle L_{\alpha}^{B}\right\rangle \subseteq B^{*}$ such that $k_{\alpha}+f \in B$. So, $k_{\alpha}=f+\left(k_{\alpha}+f\right)$ is the desired representation.

In fact, the subalgebras $B^{*}$ have a still stronger property, namely

(5) $B+\left(B^{*} \cap C\right)=C$, for all $B \leq C \leq A$.

To prove this, let $c \in C$ be given. We have just seen that $c=b+b^{*}$ for suitable $b \in B$ and $b^{*} \in B^{*}$. Noticing that $b^{*}=c+b \in C+B \subseteq C$, we see that $b^{*}$ is actually in $B^{*} \cap C$, which is all we need.

It remains to verify that

(6) $B \leq C \leq A$ implies $L_{\kappa}^{B} \supseteq L_{\kappa}^{C}$, hence $B^{*} \geq C^{*}$.

By (3), this boils down to showing that $k_{\alpha} \notin L_{\alpha+1}^{B}$ implies $k_{\alpha} \notin L_{\alpha+1}^{C}$. Assuming $k_{\alpha} \notin L_{\alpha+1}^{B}$ we get $k_{\beta_{1}}, \ldots, k_{\beta_{n}} \in L_{\alpha}^{B}$ such that

(7) $k_{\alpha}+k_{\beta_{1}}+\cdots+k_{\beta_{n}}=b \in B$.

Now we show that for each $i=1, \ldots, n$ there are $c_{i}$ and $k_{\gamma_{j}^{i}} \in L_{\alpha}^{C}$ such that

$(8, i) k_{\beta_{i}}+k_{\gamma_{1}^{i}}+\cdots+k_{\gamma_{m_{i}}^{i}}=c_{i} \in C$.

Indeed, either $k_{\beta_{i}} \in L_{\alpha}^{C}$, then we have $k_{\beta_{i}}+k_{\beta_{i}}=0$, or $k_{\beta_{i}} \notin L_{\alpha}^{C}$ and then the $k_{\gamma_{j}^{i}}$ are provided by the definition of $L_{\beta_{i}+1}^{C}$.

Adding equations $(7)$ and $(8,1), \ldots,(8, n)$, the $k_{\beta_{i}}$ cancel in pairs and we get

$$
k_{\alpha}+k_{\gamma_{1}^{1}}+\cdots+k_{\gamma_{m_{n}}^{n}}=b+c_{1}+\cdots+c_{n} .
$$

As $B \leq C$, the right-hand side is in $C$ and this shows $k_{\alpha} \notin L_{\alpha+1}^{C}$, as desired for the proof of $(6)$.

The second construction is finished. It enables us to prove implication $(a) \Rightarrow(b)$ of Theorem 1. As chains are particular cases of ramification sets, it is a special case of the assertion that every subalgebra of a pseudo-tree algebra is monotonically subalgebra-complemented. 
To prove the latter, let $C$ be a subalgebra of the pseudo-tree algebra $A$. Assertions (4), (5), and (6) show that $\left(B^{*} \cap C\right)$ is a monotonically chosen complement of $B \leq C$ with respect to $C$.

\section{How MONOTONE RETRACTIVENESS YIELDS A RAMIFICATION SET}

Here we prove the implication $(c) \Rightarrow(d)$ of Theorem 1 . Again we represent the given monotonically retractive Boolean algebra $A$ in the form $C l o p X$. For points $x_{1}, \ldots, x_{n} \in X$ we let $I\left(x_{1}, \ldots, x_{n}\right)$ denote the ideal $\left\{a \in A: x_{1} \notin a, \ldots, x_{n} \notin a\right\}$ and $C\left(x_{1}, \ldots, x_{n}\right)$ its monotonically chosen retract.

Let $\left(x_{\alpha}, y_{\alpha}\right)_{\alpha<\kappa}$ be an enumeration of $X^{2}$ and let $z$ be any fixed point of $X$. By induction, we define elements $s_{\alpha}$ of $A$. If $x_{\alpha}=y_{\alpha}$ or if these points are separated by some $s_{\beta}$ with $\beta<\alpha$, then $s_{\alpha}=\emptyset$. Otherwise we let $s_{\alpha}$ be an element of $C\left(x_{\alpha}, y_{\alpha}\right)$ that separates $x_{\alpha}$ and $y_{\alpha}$ but does not contain $z$ (actually, there is only one such $s_{\alpha}$, but this is not important for the following). Such elements exist, for, $I\left(x_{\alpha}, y_{\alpha}\right) \cup C\left(x_{\alpha}, y_{\alpha}\right)$ generates $A$. So some element of this union, $a$ say, separates the two points. By definition, $a \notin I\left(x_{\alpha}, y_{\alpha}\right)$, so $a \in C\left(x_{\alpha}, y_{\alpha}\right)$. If the first choice happens to contain $z$, it can be replaced by $X \backslash a$.

By construction, the set $S=\left\{s_{\alpha}: s_{\alpha} \neq \emptyset\right\}$ separates the points of $X$, so it generates $A=\operatorname{Clop} X$. In order to show that $S$ is a ramification set, we consider $s_{\alpha}, s_{\beta} \in S$ with $\beta<\alpha$. By symmetry, we can assume $x_{\beta} \in s_{\beta}, y_{\beta} \notin s_{\beta}$. As $s_{\beta}$ does not separate $x_{\alpha}$ and $y_{\alpha}$ (otherwise $s_{\alpha}=\emptyset \notin S$ ), there are four cases to be considered.

Case 1. $x_{\alpha}, y_{\alpha} \in s_{\beta} ; y_{\beta} \notin s_{\alpha}$. Then $s_{\alpha} \backslash s_{\beta}$ does not contain any of $x_{\alpha}, y_{\alpha}, x_{\beta}, y_{\beta}$. So, it belongs to $I\left(x_{\alpha}, y_{\alpha}, x_{\beta}, y_{\beta}\right)$. At the same time, $s_{\alpha} \backslash s_{\beta}$ is a Boolean combination of

$$
s_{\alpha}, s_{\beta} \in C\left(x_{\alpha}, y_{\alpha}\right) \cup C\left(x_{\beta}, y_{\beta}\right) \subseteq C\left(x_{\alpha}, y_{\alpha}, x_{\beta}, y_{\beta}\right),
$$

where the last inclusion comes from the assumption that the retracts are chosen in a monotone way. It follows that

$$
s_{\alpha} \backslash s_{\beta} \in I\left(x_{\alpha}, y_{\alpha}, x_{\beta}, y_{\beta}\right) \cap C\left(x_{\alpha}, y_{\alpha}, x_{\beta}, y_{\beta}\right)=\{\emptyset\},
$$

i.e. $s_{\alpha} \subseteq s_{\beta}$.

Case 2. $x_{\alpha}, y_{\alpha} \in s_{\beta} ; y_{\beta} \in s_{\alpha}$. The same argument as in case 1 yields $\left(X \backslash s_{\alpha}\right) \cap$ $\left(X \backslash s_{\beta}\right)=\emptyset$, i.e. $s_{\beta} \cup s_{\alpha}=X$. But this is impossible, because of $z$ not belonging to any element in $S$. Case 2 cannot occur.

Case 3 (resp. 4). $x_{\alpha}, y_{\alpha} \notin s_{\beta} ; x_{\beta} \notin s_{\alpha}$ (resp. $x_{\beta} \in s_{\alpha}$ ). In the same way as in case 1 we get $s_{\alpha} \cap s_{\beta}=\emptyset$ (resp. $s_{\beta} \subseteq s_{\alpha}$ ).

The desired implication and, thereby, Theorem 1 are completely proved now. Notice that the above proof used only retracts with respect to very simple ideals intersections of at most four prime ideals. An easy exercise shows that with respect to finite intersections of prime ideals every Boolean algebra always has a retract. So it is indeed the monotonicity that counts.

\section{A DiRECT PRoof of $(a) \Rightarrow(d)$}

Here we present an alternative construction showing that subalgebras of pseudotree algebras are themselves pseudo-tree algebras, i.e. generated by ramification sets. 
Let $A$ be a given pseudo-tree algebra generated by the ramification set $K$. This time it is more convenient to assume $0 \notin K$ and $1 \in K$. Assertion (1) remains in force. For $L=K$ it says that $K=K \cup\{1\}$ spans $A$ as a vector space. We can even assume that $K$ is a base in the linear algebraic sense. For, otherwise, we replace it by a subset $K^{\prime}$ which still contains 1 and is a base. Notice the important point that $K^{\prime} \subseteq K$ is automatically a ramification set. So, for the rest of this section we assume that

(9) for each non-zero $a \in A$ there is a unique non-empty finite subset $\left\{k_{1}, \ldots, k_{n}\right\}$ of $K$ such that $a=k_{1}+\cdots+k_{n}$.

We call the elements $k_{i}$ the terms of $a$ and denote the set $\left\{k_{1}, \ldots, k_{n}\right\}$ by $\tau(a)$. If $M$ is a subset of $K$, we let $\tau_{M}(a)$ abbreviate $\tau(a) \cap M$. It is reasonable to define $\tau(0)=\tau_{M}(0)=\emptyset$. Then, for each $M \subseteq K$,

(10) $\tau_{M}$ is a group homomorphism of $(A ;+)$ into $(P(M) ; \triangle)$, the power-set of $M$ under symmetric difference.

In other words, $\tau_{M}(a+b)=\left(\tau_{M}(a) \cup \tau_{M}(b)\right) \backslash\left(\tau_{M}(a) \cap \tau_{M}(b)\right)$, which is seen by adding the representations of $a$ and $b$ and cancelling the terms that occur twice.

To get the representation of $a \cdot b$ one multiplies the representations of $a$ and $b$ according to the distributive law. Afterwards repeated terms must be cancelled in pairs and possibly some zeros dropped. As $K$ is a ramification set, i.e. $k \cdot k^{\prime} \in$ $\left\{0, k, k^{\prime}\right\}$, no new terms can arise in that procedure. It follows that

(11) $\tau_{M}(a \cdot b) \subseteq \tau_{M}(a) \cup \tau_{M}(b)$.

After these preparations we embark on the proof proper. Let $B$ be a subalgebra of $A$. We want to find a ramification set generating it.

By Zorn's Lemma (use that $\tau(b)$ is finite for all $b$ ), we can fix a subset $M \subseteq K$ which is minimal with respect to the property that

(12) $\tau_{M}(b) \neq \emptyset$, for all non-zero $b \in B$.

Combined with (10) this yields the injectivity of $\tau_{M}$, i.e.

(13) $\tau_{M}(b)=\tau_{M}\left(b^{\prime}\right)$ implies $b=b^{\prime}$, for all $b, b^{\prime} \in B$.

Next we claim that

(14) for each $m \in M$ there is a unique $r_{m} \in B$ such that $\tau_{M}\left(r_{m}\right)=\{m\}$.

Indeed, by minimality, $M \backslash\{m\}$ does not satisfy (12). So some non-zero $b \in B$ has no term in $M \backslash\{m\}$. By (12), this $b$ has a term in $M$, i.e. $\tau_{M}(b)=\{m\}$. Uniqueness follows from (13).

We put $R=\left\{r_{m}: m \in M\right\}$. To see that $R$ generates $B$, consider any nonzero $b \in B$. Then $\tau_{M}(b)=\left\{m_{1}, \ldots, m_{n}\right\}$ is a non-empty, by (12), finite subset of $M$. From (10) we also get, $\tau_{M}\left(r_{m_{1}}+\cdots+r_{m_{n}}\right)=\left\{m_{1}, \ldots, m_{n}\right\}$, which yields $b=r_{m_{1}}+\cdots+r_{m_{n}}$, by (13).

To see that $R$ is a ramification set, consider $m, n \in M$. By (11), $\tau_{M}\left(r_{m} \cdot r_{n}\right)$ is one of $\{m\},\{n\}, \emptyset$, or $\{m, n\}$. In the first three cases (13) yields $r_{m} \cdot r_{n} \in\left\{r_{m}, r_{n}, 0\right\}$, as desired. The fourth case cannot occur, for, by (13), it would yield $r_{m} \cdot r_{n}=r_{m}+r_{n}$, i.e. $0=r_{m}+r_{n}+r_{m} \cdot r_{n}=r_{m} \vee r_{n}$, hence $r_{m}=r_{n}=0$, a contradiction.

\section{Topological CONSEQUences}

Here we translate Theorem 1 into the language of topology and add some more equivalent conditions. 
Theorem 2. The following conditions are equivalent for each compact and zerodimensional space $X$.

(a) $X$ is a continuous image of a compact ordered space.

(c) Retractions $f_{F}: X \rightarrow F$ can be chosen onto all non-empty closed subsets $F$ of $X$ in such a way that $F \subseteq G$ implies $f_{F}=f_{F} \circ f_{G}$.

(d) There is a ramification subset $R \subseteq C l o p X$ that separates the points of $X$.

(e) There is a continuous multiplication - on X making it a topological semilattice, i.e., associative, commutative, and idempotent, such that $x \cdot y \cdot z \in\{x \cdot y, x \cdot z\}$ for all $x, y, z \in X$.

(f) X has a cross-free closed subbase.

Recall that a retraction of a topological space $X$ onto its subset $F$ is a continuous mapping $f: X \rightarrow F$, which leaves $F$ pointwise fixed. It is well-known (cf. [14] or [13]) that a Boolean algebra is retractive iff its Stone space admits a retraction onto each non-empty closed subset.

A closed subbase is a collection $\mathcal{S}$ of closed sets such that each closed set is an intersection of finite unions of sets in $\mathcal{S}$. Possibly another expression needs explanation. A family $\mathcal{S}$ of subsets of some set $X$ is called cross-free in (some) topological contexts (cf. [7]) if $S \subseteq T$ or $T \subseteq S$ or $S \cap T=\emptyset$ or $S \cup T=X$ for all $S, T \in \mathcal{S}$.

We begin the proof of Theorem 2 with the assurance that an (advanced) exercise in Stone duality shows that $(a),(c)$, and $(d)$ are just dual to the analogously labelled conditions of Theorem 1 and, therefore, mutually equivalent.

To prove $(d) \Rightarrow(e)$, we let $R \subseteq C l o p X$ be a ramification set separating the points of $X$. As it was explained at the beginning of the previous section, we can additionally assume $\emptyset, X \in R$ and $r_{1} \triangle \ldots \triangle r_{n} \neq 0$ for pairwise distinct non-zero $r_{i} \in R$. Then

(15) for $t_{1}, \ldots, t_{n}, s_{1}, \ldots, s_{m} \in R$ the inclusion $t_{1} \cap \cdots \cap t_{n} \subseteq s_{1} \cup \cdots \cup s_{m}$ is possible only if either $t_{i} \cap t_{j}=\emptyset$ or $t_{i} \subseteq s_{j}$ for some $i, j$.

To see this, assume that $t_{i} \cap t_{j} \neq \emptyset$ for all $i, j$. Then, belonging to $R$, the $t_{i}$ are pairwise comparable and their intersection reduces to one term, $t_{1}$ say. Among the elements $s_{1}, \ldots, s_{m}$ we first drop those that do not intersect $t_{1}$ and then those that are not maximal. The remaining elements, $s_{1}, \ldots, s_{k}$ say, still cover $t_{1}$ and are pairwise disjoint. Therefore, $s_{1} \cup \cdots \cup s_{k}=s_{1} \triangle \ldots \triangle s_{k}$.

Assuming $t_{1} \not \subseteq s_{j}$ for all $j$, we get $t_{1} \supseteq s_{j}$ for all $j$. Consequently, $t_{1} \supseteq s_{1} \cup \cdots \cup$ $s_{k}=s_{1} \triangle \ldots \triangle s_{k} \supseteq t_{1}$, contradicting the additional assummption.

Next we claim that $X$ is homeomorphic to the subspace $Y$ of the Cantor cube $\{0,1\}^{R}$ consisting of all multiplicative mappings, i.e. those $y: R \rightarrow\{0,1\}$ that satisfy

(16) $y(\emptyset)=0, y(X)=1$, and $y(s \cap t)=y(s) \cdot y(t)$.

This is proved by setting $F(x)(r)=\left\{\begin{array}{ll}1, & x \in r \\ 0, & x \notin r\end{array}\right.$ and checking that $F: X \rightarrow Y \subseteq$ $\{0,1\}^{R}$ is a homeomorphism. Assertion (15) is needed to get surjectivity.

Now, $Y$ is closed under pointwise multiplication in $\{0,1\}^{R}$, which is a semilattice. It remains to verify that for all $x, y, z \in Y$ either $x \cdot y \cdot z=x \cdot y$ or $x \cdot y \cdot z=x \cdot z$. If that were not true, we could find $s$ and $t$ in $R$ such that

$$
x(s)=1, y(s)=1, z(s)=0, \quad \text { and } \quad x(t)=1, y(t)=0, z(t)=1 .
$$


But then, by (16), neither $s \cap t=\emptyset$ (because of $x$ ) nor $s \subseteq t$ (because of $y$ ) nor $t \subseteq s$ (because of $z$ ). This contradicts the ramification property of $R$.

Next we prove $(e) \Rightarrow(d)$. Let $(X, \cdot)$ be a topological semilattice such that $x \cdot y \cdot z \in\{x \cdot y, x \cdot z\}$ for all $x, y, z \in X$. Consider the collection $R \subseteq \operatorname{Clop} X$ consisting of all

$$
r(\varphi, h)=\{x \in X: \varphi(x) * h=h\},
$$

where $\varphi: X \rightarrow H$ is a continuous homomorphism into some finite semilattice $(H, *)$ and $h \in H$. To see that $R$ separates the points of $X$, we consider $x \neq y$. Numakura's Theorem from [11] says that every compact and zero-dimensional semigroup is profinite. It follows that $x$ and $y$ can be separated by a continuous homomorphism $\varphi: X \rightarrow H$ onto a finite semilattice. Without loss, $\varphi(x) * \varphi(y) \neq \varphi(x)$, hence $x \in r(\varphi, \varphi(x)) \not \supset y$.

To see that $R$ is a ramification set, we consider homomorphisms $\varphi: X \rightarrow H$ and $\psi: X \rightarrow K$ and elements $h \in H$ and $k \in K$. Aiming at a contradiction we assume

$$
r(\varphi, h) \nsubseteq r(\psi, k) \quad \text { and } \quad r(\psi, k) \nsubseteq r(\varphi, h) \quad \text { and } \quad r(\psi, k) \cap r(\varphi, h) \neq \emptyset .
$$

This yields points $x, y, z \in X$ such that $\varphi(y) * h=h, \psi(y) * k \neq k$ and $\psi(z) * k=$ $k, \varphi(z) * h \neq h \quad$ and $\quad \varphi(x) * h=h, \psi(x) * k=k$.

Then, using that $H$ is a semilattice,

$\varphi(x \cdot y \cdot z) * h=\varphi(x) * \varphi(y) * \varphi(z) * h^{3}=(\varphi(x) * h) *(\varphi(y) * h) * \varphi(z) * h=h * h * \varphi(z) * h \neq h$

and, analogously, $\psi(x \cdot y \cdot z) * k \neq k, \varphi(x \cdot y) * h=h$, and $\psi(x \cdot z) * k=k$. Together these values contradict $x \cdot y \cdot z \in\{x \cdot y, x \cdot z\}$, which ends the proof of $(e) \Rightarrow(d)$.

The implication $(d) \Rightarrow(f)$ is proved by putting $\mathcal{S}=R \cup\{X \backslash r: r \in R\}$. The proof of $(f) \Rightarrow(d)$ is more interesting. Let $\mathcal{S}$ be a cross-free closed subbase of the topology of $X$. The principal difficulty lies in showing that

(17) $\mathcal{S} \cap$ Clop $X$ separates the points of $X$.

To do so, we let $x \neq y$ be given and choose $a \in C l o p X$ such that $x \in a \not \supset y$. As $a$ is closed, it can be represented as an intersection of finite unions $\left(s_{1}^{i} \cup \ldots \cup s_{m_{i}}^{i}\right)$ of elements of $\mathcal{S}$. As $a$ is also open, a finite intersection

$$
a=\bigcap_{i=1}^{n}\left(s_{1}^{i} \cup \ldots \cup s_{m_{i}}^{i}\right)
$$

is sufficient (by compactness). We can further assume that $s_{p}^{i} \cap s_{q}^{i}=\emptyset$ for all $i$ and $1 \leq p<q \leq m_{i}$. Indeed, if one of $s_{p}^{i}$ or $s_{q}^{i}$ is contained in the other, it may be dropped from $s_{1}^{i} \cup \ldots \cup s_{m_{i}}^{i}$. If $s_{p}^{i} \cup s_{q}^{i}=X$, then the whole union equals $X$ and may be dropped from the intersection $(a \neq X !)$. As $\mathcal{S}$ is cross-free, the purged representation of $a$ satisfies the additional assumption.

Using the distributivity of the set-theoretic operations we rewrite

$$
a=\bigcup_{f} \bigcap_{i=1}^{n} s_{f(i)}^{i}
$$

where $f$ runs over all mappings $\{1, \ldots, n\} \rightarrow\left\{1, \ldots, \max m_{i}\right\}$ such that $f(i) \leq m_{i}$ for all $i$. 
The sets $\bigcap_{i=1}^{n} s_{f(i)}^{i}$ are, obviously, closed. They are also pairwise disjoint, for $f \neq g$ means $f(j) \neq g(j)$ for some $j$, hence

$$
\bigcap_{i=1}^{n} s_{f(i)}^{i} \cap \bigcap_{i=1}^{n} s_{g(i)}^{i} \subseteq s_{f(j)}^{j} \cap s_{g(j)}^{j}=\emptyset .
$$

As their union $a$ is open, all $\bigcap_{i=1}^{n} s_{f(i)}^{i}$ are clopen and one of them contains $x$ but not $y$. To simplify notation, we now consider a clopen intersection $b=s_{1} \cap \cdots \cap s_{n}$ of (without loss) pairwise incomparable elements of $\mathcal{S}$, such that $x \in b \not \supset y$. There is no loss in further assuming that $x \in s_{1} \not \supset y$. Our aim is to show that $s_{1}$ is actually clopen. For $j=2, \ldots, n$ we must have $s_{1} \cup s_{j}=X$, for $x \in s_{1} \cap s_{j} \neq \emptyset$ and comparability is excluded by assumption. It follows that $X=s_{1} \cup\left(s_{2} \cap \cdots \cap s_{n}\right)$, hence, denoting $\left(s_{2} \cap \cdots \cap s_{n}\right)$ by $c$,

$$
X=s_{1} \cup c=\left[s_{1} \backslash\left(s_{1} \cap c\right)\right] \cup\left[s_{1} \cap c\right] \cup\left[c \backslash\left(s_{1} \cap c\right)\right] .
$$

Given that $s_{1} \cap c=b$ is clopen, this is a decomposition of $X$ into three disjoint closed sets. It follows that they are all clopen and so is

$$
s_{1}=\left[s_{1} \backslash\left(s_{1} \cap c\right)\right] \cup\left[s_{1} \cap c\right],
$$

as was to be shown. Now, that (17) is proved, an easy verification shows that

$$
\mathcal{R}=\{s: z \notin s \in \mathcal{S} \cap \text { Clop } X\} \cup\{X \backslash s: z \in s \in \mathcal{S} \cap \text { Clop } X\}
$$

is the desired ramification set separating the points of $X$. Here (as in section 2) $z$ is any point of $X$ chosen in advance.

We finish by mentioning to the initiated reader that in [7, Theorem 6.6] J. van Mill and E. Wattel prove condition $(f)$ of Theorem 2 for arbitrary compact spaces equivalent to

(g) $X$ can be embedded into a dendron.

This explains the occurrence of dendrons in Purisch's proof of $(a) \Leftrightarrow(d)$ mentioned in the introduction.

\section{REFERENCES}

[1] Bonnet, R. Subalgebras. Chapter 10 in vol. 2 of: J. D. Monk (ed.) Handbook of Boolean algebras. North-Holland, Amsterdam 1989

[2] Bonnet, R., Rubin, M., Si-Kaddour, H. Generating sets of superatomic subalgebras of interval algebras. Submitted to Proc. Amer. Math. Soc.

[3] van Douwen, E. K. Small tree algebras with nontree subalgebras. Topology and its Applications 51(1993), 173-181. MR 94m:06014

[4] Koppelberg, S. General theory of Boolean algebras. vol. 1 of: J. D. Monk (ed.) Handbook of Boolean algebras. North-Holland, Amsterdam 1989

[5] Koppelberg, S., Monk, J. D. Pseudo-trees and Boolean algebras. Order 8(1992), 359-374. MR 93i:06006

[6] Koppelberg, S. Counterexamples in minimally generated Boolean algebras. Acta Univ. Carolinae - Math. et Physica, 29(1988), 27-36. MR 90a:06014

[7] van Mill, J., Wattel, E. Dendrons. 59-82 in: H. R. Bennet, D. J. Lutzer (eds.), Topology and order structures, part I, Mathematical Centre Tracts vol. 142, Amsterdam 1981.

[8] Monk, J. D. Notes on pseudo-tree algebras. informal notes, September 1994.

[9] Mostowski, A. and A. Tarski Boolesche Ringe mit geordneter Basis. Fundamenta Mathematicae 32(1939), 69-86.

[10] Nikiel, J. Orderability properties of a zero-dimensional space which is a continuous image of an ordered compactum. Topology and its Applications 31(1989), 269-276. MR 91g:54041

[11] Numakura, K. Theorems on compact totally disconnected semigroups and lattices. Proc. Amer. Math. Soc. 8(1957), 623-636. MR 19:290d 
[12] Purisch, S. In Topology proceedings 17(1994), Problem section, p. 412.

[13] Rao, K.P.S. Bhaskara and M. Bhaskara Rao On the lattice of subalgebras of a Boolean algebra. Czechoslovak Math. Journal 29(1979), 530-545. MR 80j:06017

[14] Rotman, B. Boolean algebras with ordered bases. Fundamenta Mathematicae 75(1972), 187197. MR 46:1671

[15] Rubin, M. A Boolean algebra with few subalgebras, interval Boolean algebras and retractiveness. Trans. Amer. Math. Soc. 278(1983), 65-89. MR 85a:06024

[16] Shelah, S. On uncountable Boolean algebras with no uncountable pairwise comparable or incomparable sets of elements. Notre Dame Journal of Formal Logic, 22(1981), 301-308. MR 83d:03060

Freie Universität Berlin, 2. Mathematisches Institut, Arnimallee 3, D - 141915 Berlin, Germany

E-mail address: heindorf@math.fu-berlin.de 